\title{
Breaking Down Barriers: Accessibility in Planetary Science
}

\section{Primary Author}

Jennifer L. Piatek, Dept. of Geological Sciences, Central Connecticut State University, New Britain, CT; piatekjel@ccsu.edu

\section{Co-Authors}

Kathleen E. Vander Kaaden, Jacobs/NASA JSC; kathleen.e.vanderkaaden@nasa.gov

Timothy A. Goudge, Dept. of Geological Sciences, The University of Texas at Austin, Austin, TX; tgoudge@jsg.utexas.edu

Jamie L. Molaro, Planetary Science Institute, Tucson, AZ; imolaro@psi.edu

Moses P. Milazzo, Other Orb LLC, Flagstaff, AZ; moses@otherorb.net

\section{Co-Signers}

This white paper is submitted as part of a collaborative effort organized by the Equity, Diversity, and Inclusion Working Group (EDIWG), a cross Assessment Group (AG) committee. In addition, our content and recommendations have been endorsed by the International Association for Geoscience Diversity (IAGD, http://www.theiagd.org).

As of September 15th, this paper had $~ 75$ co-signers: the updated list may be viewed at: https://docs.google.com/spreadsheets/d/1nJ3ir coeYcMIXEVq9sw-h-jUE1290WcYwReJ Rxw7y4/edit?usp=sharing

We are still accepting new cosigners: please add your name to co-sign via the form at https://forms.gle/43XTNaws1VowUUva5. 


\subsection{INTRODUCTION}

Planetary science represents a diverse set of disciplines dedicated to exploring the limits of the solar system and beyond: we build on the foundational concepts of physics, chemistry, geology, and biology to learn more about the worlds in our universe and innovate from the newest ideas in engineering to enable that exploration. We are missing, however, the voices from parts of our community who also strive to study these remote locales but are required to navigate barriers erected by inaccessible locations in our scientific workplaces, field sites and conferences/workshops; and negative perceptions of how to address those issues. NASA's "A Vision for Science Excellence" identifies a goal to "[i]ncrease the diversity of thought and backgrounds represented across the entire SMD portfolio through a more inclusive and accessible environment" .

\section{The goal of this white paper is to address how and why accessibility matters to planetary science and those who have chosen this field as a career, and what can be done to overcome some of the many barriers faced by individuals with accessibility concerns.}

\subsection{BACKGROUND}

Demographics for scientists with disabilities can be difficult to compare, as different survey tools utilize various definitions of disability, and this demographic information is not always collected (e.g. past surveys of the membership of the Division for Planetary Sciences ${ }^{2}$ ). Statistics aggregated by the National Science Foundation (NSF), including the Survey of Earned Doctorates ${ }^{3}$, the Survey of Doctorate Recipients ${ }^{4}$, and the National Survey of College Graduates ${ }^{5}$ suggest that individuals with disabilities are underrepresented in the physical sciences and engineering, representing only 10\% of those with a Bachelor's degree or higher employed in these sectors, and represent only $5 \%$ of those employed in physics and astronomy. These NSF studies utilize similar criteria to the American Community Survey, the latest of which numerates $12.6 \%$ of the U.S. population as considered "disabled" - that is, an individual who identifies as having "moderate" or "severe" difficulty, or is "unable to do" tasks such as seeing (with glasses), hearing (with hearing aid), walking without assistance, lifting 10 pounds, or concentrating, remembering, or making decisions, although this definition has changed since 2010. In addition to under presentment in science and engineering employment, the statistics from these NSF studies also suggest that doctoral students with disabilities are more likely to self/family fund their graduate studies and are less likely to be funded as a research assistant or via a

\footnotetext{
${ }^{1}$ https://science.nasa.gov/science-red/s3fs-public/atoms/files/2020-2024_Science.pdf

${ }^{2}$ Available at https://dps.aas.org/reports

${ }^{3}$ https://www.nsf.gov/statistics/srvydoctorates/

${ }^{4}$ https://www.nsf.gov/statistics/srvydoctoratework/

${ }^{5}$ https://www.nsf.gov/statistics/2020/nsf20300/

${ }^{6}$ https://www.census.gov/programs-surveys/acs
} 
fellowship, and if employed in the sciences/engineering after graduation are likely to earn $5 \%$ less than their peers without disabilities. Although not directly comparable due to changes in the definition of "disabled" used in these metrics, the number of doctorates awarded to students with disabilities increased by only $0.009 \%$ between 1985 and 2010, despite the fact that this time period includes the passage of federal legislation designed to increase educational opportunities for disabled Americans (the Americans with Disabilities Act, or ADA, and the Individuals with Disabilities Education Act; Booksh, 2017). The effect of inaccessibility and strategies for creating more inclusive workplaces within the astronomy and physics workforce were also a factor in the compilation of the latest Astronomy decadal survey (e.g. Aarnio et al., 2019). In addition, the difficulties in interpreting these data are compounded by the lack of representation for seemingly "invisible" disabilities that are not necessarily captured in the classical definition including, but not limited to, medical challenges such as epilepsy, severe dietary allergies, chronic illnesses, neurodivergence, and mental health conditions. In order to promote inclusion of diverse voices, it is necessary to both recruit and retain the individuals who have those diverse voices. In this white paper, we will specifically focus on the key factors affecting accessibility. We will also provide recommendations to promote a more accessible and inclusive planetary science community.

The recommendations included in this document represent strategies for both attracting students to planetary science and retaining those planetary scientists within the field as they progress through their career.

\subsection{Key Factors Affecting Accessibility}

One aspect of planetary science careers that may lead to the disparities discussed here is the perception of the accessibility of the field for individuals with disabilities. A significant fraction of planetary scientists come from geoscience backgrounds ${ }^{7}$, a career path that can be perceived as inaccessible. Atchison and Libarkin (2016) conducted a survey of attendees at a large national geoscience conference and identified three potential barriers to access:

\section{1) Perceptual Barriers}

- the assumption that people with disabilities cannot complete tasks relevant to geoscience careers

\section{2) Training Barriers}

- exclusion of those who have difficulty with skills perceived "critical" to the field even if those skills are tangential to all career paths

\section{3) Community-Based Barriers}

- lack of available accommodations in classroom and professional settings

\footnotetext{
${ }^{7}$ http://dps.aas.org/reports
} 
Of particular relevance is the "training barrier" presented by field and/or lab work: often, geoscience degrees (including those with planetary science focuses) require that students complete coursework that includes training in the field or laboratory that is not necessarily accessible to all students. Carabajal et al. (2017) noted that when accommodations in the lab or field are available and all students are able to participate, students with disabilities are as successful as their peers without disabilities: but often these accommodations are limited to "alternative" assignments that exclude those who must complete them from the social aspect of the learning experience. They concluded by stating that "[w]e must end the promotion of rigorous physical activity as the only way to learn geoscience skills and knowledge. Doing so will enable every geoscience student, instructor, researcher, and practitioner to contribute his or her own experiences, according to his or her level of ability, in an inclusive community of learning." Furthermore, Stokes et al. (2019) found that practices to broaden accessibility to geoscience fieldwork benefit all involved students through improved pedagogical design.

\section{Planetary scientists work with some of the most inaccessible field sites and have access to some of the most cutting-edge technologies in the lab; we should also be applying our skills to making field sites and lab work more accessible to everyone. Additional recommendations regarding accessibility of field work are discussed by Richardson et al. (2020).}

Another significant aspect of science careers that often present barriers to access are in-person meetings such as scientific conferences, team meetings, or review panels (e.g. experiences related by Sohn, 2019). These events provide critical opportunities to share science and network with others to develop more ambitious and innovative research programs. The travel related to these events, however, can exclude portions of our community, particularly those who must limit travel due to impact of disabilities or other medical conditions (temporary or permanent), or simply due to the required expense and time. Accessible airline seats are often limited or restricted to higher fares (e.g. pre-selected seats or first/business class), accessible taxis or rental vehicles may not be available at all destinations, air travel may require extra planning due to time needed to board/disembark planes, and some people may need to travel with personal assistants. Many of these incur additional costs that fall outside the reimbursement limits set by institutions, and thus are borne directly by the scientist themselves.

Although most conferences take place in ADA compliant facilities, these locations can still present barriers to attendance. For example:

- Conference centers may lack Braille signs.

- Conference rooms are often set up without adequate room for mobility devices.

- Events lack options to assist attendees who are unable to hear conference proceedings (including individual talks, plenaries, and workshops).

- Conference podiums often require navigation of stairs to present. 
- Poster sessions that require standing for long periods without available chairs.

- Conference centers that are crowded with attendees can be difficult or impossible to navigate for those who are neurodivergent or suffer from anxiety.

- Conference snacks often lack appropriate allergy warnings or pertinent handling information.

- Conference hotels that are a far distance from the conference center, and/or conference hotel rates that are above the allowed per diem.

An informal survey of planetary scientists suggests that a significant fraction of the community experiences barriers to conference travel (Piatek and Ostrach, 2020). Over half of the respondents to the cited survey (58\%) indicated that they had opted to not travel to some conferences because they felt those meetings were inaccessible. Some of the specific issues highlighted in this survey included meeting cost $(80 \%$ had chosen not to travel due the high expense and/or lack of funding), meeting schedules that were not published in time to make travel plans or lacked sufficient break time, venues that were perceived as unsafe or that lacked nearby hotels and/or appropriate food options, lack of seating at events such as poster sessions, and presentations that were difficult to understand due to poor room layout, lighting, or a lack of audio solutions.

Assuring that all professional gatherings, such as conferences, are accessible for all is key for maintaining workforce diversity and presenting an inclusive work environment.

4.0 ReCOMMENDATIONS

Although potentially receiving additional attention today, this is by no means a new topic nor challenge. Numerous groups have already put in a lot of work to provide support in order to increase accessibility across the sciences. We strongly recommend that planetary science departments, research centers, national laboratories, federal facilities, etc., adopt relevant principles and recommendations from appropriate advocacy groups, including the Working Group on Accessibility and Disability of the American Astronomical Society ${ }^{8}$ and the International Association for Geoscience Diversity ${ }^{9}$.

Promote inclusive practices at all sponsored conferences, team meetings, panels, and other professional gatherings.

Require that all meetings, in-person or virtual, publish accessibility information that includes a point of contact for accessibility concerns. Develop a request format such

\footnotetext{
8 http://wgad.aas.org

${ }^{9}$ http://www.theiagd.org
} 
that attendees are able to express concerns about accessibility anonymously, for those who do not wish to make their requests for accommodation public.

Require that proposed workshops, meetings, conferences, etc., include an accessibility plan (ADA compliance, Section 508 compliance, etc.) as part of their technical merit section and include people with experience in accessibility in the review of such proposals. Include an after-action-review of workshops, meetings, conferences, etc., that considers how successful the event met its accessibility obligations.

Require that all meetings, in-person or virtual, provide a schedule of events in a timely fashion so attendees can adequately plan.

Recommend to organizers that events, regardless of size, adhere to best practices for accessible meeting design, such as (but not limited to) those provided by:

- SciAccess ${ }^{10}$

- The ADA National Network ${ }^{11}$

- Informal guidance from the Union of Concerned Scientists ${ }^{12}$.

Included in these design recommendations are designated quiet rooms and parents' rooms, as well as appropriate accessible design for poster and oral sessions to accommodate attendees with limited mobility and signage for those with visual impairments.

Recommend that all conferences include options for virtual attendance, including the ability to participate in events virtually even if attending the meeting in person.

Recommend that events include access to technologies designed to assist those with hearing impairments, such as sign language interpreters, live captioners, and/or hearing loops. Virtual meetings should include captioning, preferably via a live captioner as current auto-caption software is often inaccurate with highly technical language.

Recommend that any food provided by the event is properly labelled with allergy and handling information.

Recommend that the NASA Guidebook for Proposers provide direct guidance to proposing institutions regarding the need to include additional travel costs such as higher air fares and/or larger rental vehicles in proposal budgets when necessary for safe and accessible travel, and that funding organizations be explicit that travel costs required for accessibility reasons are reimbursable when so budgeted.

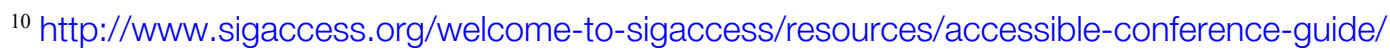

${ }^{11} \mathrm{https}$ //adata.org/guide/planning-guide-making-temporary-events-accessible-people-disabilities

${ }^{12} \mathrm{https}: / /$ blog.ucsusa.org/science-blogger/how-to-make-professional-conferences-more-accessible-for-disa bled-people-guidance-from-actual-disabled-scientists
} 


\section{Promote development of an inclusive scientific community by prioritizing mentoring for students and early career scientists.}

Require training for all educators, mentors, professionals, etc. that enables them to 1) recognize that disabilities in students/mentees/colleagues may not always be visible, 2) provide support for all students/mentees/colleagues, and 3) modify existing curriculum/programs to ensure inclusion of students/mentees/colleagues that might otherwise not have access.

Recommend that proposals requesting funding for students and/or postdocs include a description of mentoring activities, including but not necessarily limited to work/life balance issues, flexible work schedules (including options for telework), and flexible graduation schedules; and that proposals demonstrate that funded students/postdocs will have access to ergonomic and accessible workspaces.

Recommend that all internship and research experiences for students include mentoring: providing information about career opportunities, how to get into grad school, what is expected in grad school, what is a postdoc and why do you need one?

Support virtual internship opportunities for students who are unable to live away from home for 6-10 weeks, even with travel funding and/or stipends. Supervisors for these experiences should receive additional training on best practices for remote mentoring.

\section{Promote accessibility in planetary science at all educational and professional levels.}

Recommend that all funded lab facilities not only adhere to ADA requirements, but go beyond where possible, recognizing that the ADA requirements represent a minimum level of accessibility, not a maximum limit.

Recommend that programs that incorporate innovative technology and project design include specific objectives to include work that addresses accessibility concerns, particularly those relevant to lab and field work for terrestrial analogs. For example, the Planetary Science and Technology from Analog Research (PSTAR) program could specifically call out accessibility as a new, fourth program objective, from which proposed projects must address two.

Support initiatives to develop educational materials using principles of universal design ${ }^{13}$ which moves the task of making content accessible to the designer rather than the end user.

Develop programs to increase accessibility of field-based and/or lab-based coursework, often required for students pursuing degrees in planetary science, and

\footnotetext{
${ }^{13} \mathrm{http}: / /$ udlguidelines.cast.org/
} 
apply the results of these programs to promoting continued access to lab and field work for professional planetary scientists.

\begin{abstract}
Although far from comprehensive, we believe the recommendations listed above provide a template for NASA and related organizations, research centers, and universities to make their programs more accessible and a pathway to continue to find new and innovative ways to make these environments where people with disabilities can thrive and make innovative contributions to planetary science.
\end{abstract}

\title{
5.0 REFERENCES
}

Aarnio, Alicia, Nicholas Murphy, Karen Knierman, Wanda Diaz Merced, Alan Strauss, Sarah Tuttle, Jacqueline Monkiewicz, et al. 2019. "Astro2020 APC White Paper: Accessible Astronomy: Policies, Practices, and Strategies to Increase Participation of Astronomers with Disabilities," July, 1-16. https://arxiv.org/abs/1907.04943.

Atchison, Christopher L., and Julie C. Libarkin. 2016. "Professionally Held Perceptions about the Accessibility of the Geosciences." Geosphere 12 (4): 1154-65.

https://doi.org/10.1130/GES01264.1.

Booksh, Karl S. 2017. "Why Are There so Few Doctorates with Disabilities in Chemistry? Thoughts and Reflections." In , 195-203. https://doi.org/10.1021/bk-2017-1256.ch018.

Carabajal, Ivan G., Anita M. Marshall, and Christopher L. Atchison. 2017. "A Synthesis of Instructional Strategies in Geoscience Education Literature That Address Barriers to Inclusion for Students With Disabilities." Journal of Geoscience Education 65 (4): 531-41. https://doi.org/10.5408/16-211.1.

Piatek, J.L. and L.R. Ostrach. 2020. "Sharing Science with the Widest Audience: Making Scientific Conferences Accessible." Lunar and Planetary Science Conference 51, abstract \#2406. (also available as an e-poster ${ }^{14}$ ).

Richardson, J., Whelley, N., and P. Whelley. 2020. "Building Safer and More Inclusive Field Experiences in support of Planetary Science". A White Paper for NASA's Planetary Science and Astrobiology Decadal Survey 2023-2032.

Sohn, Emily. 2019. "Ways to Make Meetings Accessible." Nature 576 (7787): S74-75. https://doi.org/10.1038/d41586-019-03852-2.

Stokes, Alison, Anthony D. Feig, Christopher L. Atchison, and Brett Gilley. 2019. "Making geoscience fieldwork inclusive and accessible for students with disabilities." Geosphere 15 (6): 1809-1825. https://doi.org/10.1130/GES02006.1.

\footnotetext{
${ }^{14}$ https://www.hou.usra.edu/meetings//psc2020/eposterindex.cfm/eposter/2406.pdf
} 